\title{
MODELO BASADO EN SIG PARA EL ESTUDIO DE LA MORTALIDAD POR ENFERMEDAD CEREBRO-VASCULAR
}

\author{
Carlos Alberto Tangarife Villa \\ Universidad de Antioquia - UdeA \\ Especialista en Ciencias Electrónicas $e$ \\ informática área: bases de datos \\ ctangar@gmail.com
}

\author{
Libardo Londoño Ciro \\ Universidad San Buenaventura seccional Medellín \\ Especialista en Sistemas de Información \\ Geográfica \\ libaranto@une.net.co
}

(Tipo de Artículo: Investigación. Recibido el 22/03/2013 Aprobado el 28/05/2013)

\begin{abstract}
RESUMEN
En los últimos 15 años el estudio para una de las tres primeras causas de muerte a nivel mundial -enfermedad cerebrovascular (ECV) - se caracteriza por utilizar el enfoque epidemiológico tradicional y sus técnicas de análisis estadístico bivariado y multivariado orientadas a identificar su composición según variables de persona y explorar relaciones con sus determinantes pero sin impactar significativamente su ocurrencia, sin embargo, en el presente artículo se identifica como alternativa de análisis la modelación espacio-temporal con SIG a niveles de desagregación detallados; en este sentido el análisis comparativo de los modelos utilizados en los estudios conocidos, desvela el uso de datos y técnicas de análisis espacial básicas para identificar patrones como los evidenciados en los cinturones de diabetes y ECV en USA o el clúster colombiano de enfermedades del aparato circulatorio identificado en la región andina que sugiere un comportamiento similar para la ECV. El panorama anterior demanda la combinación de enfoques con énfasis en sus técnicas de análisis avanzadas análisis multi-criterio, kriging, interpolación, correlación y regresión espacial- soportadas con SIG y por lo tanto, como contribución final se propone un modelo integrado dirigido a impactar positivamente la mortalidad por ECV.
\end{abstract}

\section{Palabras clave}

Análisis espacial, accidente cerebrovascular, Sistemas de Información Geográfica, patrones de mortalidad, enfermedad cerebrovascular, ECV.

\section{SPATIO-TEMPORAL APPROACH FOR MODELING THE MORTALITY BY CEREBROVASCULAR DISEASE}

\begin{abstract}
During the last 15 years the approach to mortality regarding to one of the three main causes of death worldwide, cerebrovascular disease (CVD), has been characterized by the use of traditional epidemiological approach that incorporates bivariate and multivariate statistical analysis techniques aimed to identify its composition based on individual variables and exploring relationships with its determinants without significantly affect its occurrence, however, in this article is identified as an alternative analysis the spatio temporal modeling with GIS having detailed disaggregation levels; therefore, the comparative analysis of the models used in the known studies, revealed the use of basic data and basic spatial analysis techniques which contribute to produce geographical knowledge and identifying patterns as those evidenced in the diabetes and CVD belts in USA or the colombian cluster identified for circulatory system diseases in the Andean Region, suggesting a similar behavior for CVD. The above scenario requires combining approaches focused on advanced analysis techniques, multi-criteria analysis, kriging, interpolation, spatial-correlation and regression supplied GIS and therefore, we propose a model with an integrated approach aimed to positively affect CVD mortality.
\end{abstract}

\section{Keywords}

Spatial analysis, stroke, geographic information systems, mortality patterns, cerebrovascular diseases, CVD. 


\section{APPROCHE SPATIO-TEMPOREL POUR LA MODÉLISATION DE LA MORTALITÉ PAR MALADIE CÉRÉBRO-VASCULAIRE}

\section{RÉSUMÉ}

Dans les derniers 15 ans l'approche de la mortalité pour une des trois principales causes de mort dans le monde, maladie cérébro-vasculaire (MCV), s'est caractérisé par la utilisation de l'approximation épidémiologique traditionnelle qu'incorpore des techniques d'analyse statistique bivariée et multivariée orientes vers l'identification de sa composition d'après variables de personne et d'explorer des relations avec ses déterminants sans affecter d'une manière significative s'apparition, cependant, dans cet article on identifier comme alternative d'analyse comparatif des modèles spatio-temporel avec SIG sur niveaux de désagrégation détaillés; en ce sens le analyse comparatif des modèles utilisés dans les études connus, a dévoilé l'usage de données et techniques de base d'analyse spatial qui ont contribué pour créer de la connaissance géographique et d'identifier des patrons comme ces mis en évidence dans les ceintures de diabète et maladie cérébro-vasculaire aux états unis ou le cluster colombien de maladies de l'appareil circulatoire identifie dans la région andine, qui nous suggère un comportement similaire pour la MCV. Le horizon qu'on a décrit requiert la combinaison d'approches avec emphase sur des techniques avancées d'analyse - analyse multicritère, kriging, interpolation, corrélation et régression spatial- et par conséquent, on propose un modèle d'approche intégré dirigé vers affecter de manière positive la mortalité par MCV.

\section{Mots-clés}

Analyse spatial, accident cérébro-vasculaire, systèmes d'information géographique, patrons de mortalité, maladie cérébrovasculaire, $M C V$ 


\section{INTRODUCCIÓN}

El estudio de la mortalidad como un fenómeno demográfico es fundamental para entender la dinámica poblacional de una localidad, conocer su estado de salud y contribuir a disminuir las muertes prematuras, además se convierte en un insumo fundamental para apoyar el establecimiento de políticas, consolidar los procesos de planificación y orientar las acciones en salud; a pesar de que la mortalidad es un evento seguro su comportamiento difiere a nivel continental, regional, local e inclusive dentro de grupos particulares de población según factores individuales y culturales, por lo tanto, se hace necesario abordar esta desde diferentes puntos de vista y de acuerdo con diferentes niveles de desagregación geográfica y de agrupación de causas de muerte.

La mortalidad se puede analizar para un grupo de causas o una causa en particular. Según la Organización Mundial de la salud-OMS para sus países miembros en el 2008 las dos primeras causas de muerte corresponden en el primer lugar, para las muertes por enfermedad isquémica del corazón $12.8 \%$ $y$ en el segundo lugar, las ocasionadas por accidentes cerebro-vasculares acompañadas de otras enfermedades cerebro-vasculares (ECV) con un 10.8\% del total de muertes registradas, el panorama anterior ubica la mortalidad por ECV en un lugar de atención prioritaria y la convierte en un objeto de estudio fundamental; más aún, cuando de acuerdo con las proyecciones de la OMS se espera que para el año 2020 las muertes por ECV aumenten a un 11.5\% con respecto al total de muertes [1]. Dada la magnitud del problema y por ser un grupo de enfermedades con factores de riesgo modificables cuya corrección potencialmente prevendría la aparición de la enfermedad, es necesario analizar a profundidad las características de este grupo de enfermedades en cuanto la distribución de factores de riesgo en la población, incidencia, letalidad y mortalidad.

A nivel mundial se realizan diferentes estudios sobre el comportamiento de la mortalidad causada por ECV con respecto a su composición por edad, sexo y según unidades geográficas subnacionales [2], [3], [4], [5], [6], pero muy pocos exploran sus relaciones o patrones espacio-temporales mediante el uso de SIG a niveles de desagregación más detallados como los municipios en el caso Colombiano [7].

En el caso particular Colombiano, con la mejora de los sistemas de registro de las estadísticas vitales desde la década de los años 90 , se potenciaron las iniciativas de investigación a diferentes niveles de desagregación [8]. Específicamente con respecto a la mortalidad por ECV se adelantan iniciativas a niveles regionales, departamentales y eventualmente para un municipio en particular, sin embargo, a la fecha los estudios conocidos sobre mortalidad por ECV no consideran caracterizaciones como la descomposición de la mortalidad según municipio durante un período prolongado de tiempo, a la vez que tampoco se relaciona la incidencia de las causas de mortalidad por ECV con respecto a sus determinantes y mucho menos se establecen perfiles de causas por municipio o identifican patrones de comportamiento espaciotemporales para dicha enfermedad.

De acuerdo con el panorama anterior es necesario preparar el terreno para profundizar en el estudio de la ECV a niveles de desagregación detallados, en tal sentido en el presente artículo se describen los dos enfoques metodológicos utilizados para generar conocimiento sobre la mortalidad por enfermedad cerebro-vascular-ECV -enfoque tradicional y enfoque espacio-temporal- además, se comparan los modelos utilizados por el segundo enfoque de acuerdo con sus componentes principales -entradas, herramientas de análisis y salidas- y se propone un modelo general para abordar la mortalidad por ECV que integra ambos enfoques.

\section{MODELACIÓN Y ESTUDIO DE LA MORTALIDAD POR ECV 1997-2012}

Según la OMS para el 2008 se presume un aumento de la mortalidad por ECV para en el año 2020 [9], este panorama permite evidenciar que este tipo de enfermedades deben ser una de las prioridades de investigación en los próximos años debido a su magnitud y a los efectos que pueden tener en la estrategia de atención primaria en salud vigente.

Desde un punto de vista geográfico el tema de la salud de una población se relaciona de alguna manera con las características del entorno inmediato, por lo tanto, el estudio de la distribución espacio temporal de las enfermedades y de la mortalidad que ocasionan es una aproximación básica para identificar sus posibles causas, determinar su comportamiento e impactar positivamente su ocurrencia.

Las aplicaciones de la cartografía temática, es decir, el uso de los mapas temáticos comenzó alrededor del año 1800 con usos ambientales y sociales; en esta misma época también se comenzaron a mapear enfermedades y los primeros mapas referidos en la literatura mencionan eventos infecciosos como los de fiebre amarilla en Estados Unidos en 1798 y el de cólera en Londres en 1855 [10]. Haviland en 1875 fue pionero en la construcción de mapas para enfermedades crónicas, principalmente enfermedades del corazón y cáncer en Inglaterra y Gales [11] además, fue el primero en calcular tasas de mortalidad brutas entre 1851 y 1860 . Después de los años 60 y hasta la época actual, impulsados principalmente por el desarrollo tecnológico un número importante de autores desarrollan e implementan numerosos métodos de utilidad para el mapeo de enfermedades y 
por consiguiente generan conocimiento geográfico fundamental para explicar diferentes fenómenos en salud [11].

Históricamente, los eventos de salud se registran de forma agregada para grandes áreas geográficas determinadas administrativamente como son continentes, países, departamentos, estados o provincias y con restricciones para áreas detalladas como los municipios o en algunos casos los condados - parroquias; la agregación de grandes áreas geográficas para la geo-referenciación de los eventos se realiza de forma generalizada en la mayor parte de los países, pero su principal desventaja es que los problemas específicos que evidencian posibles agregaciones geográficas significativas (clusters) 0 en general patrones de comportamiento espacio-temporal a niveles detallados no pueden ser investigados a menos que sus límites geográficos coincidan con las unidades espaciales utilizadas para su georeferenciados[12].

En la actualidad existen tres hechos que cambian la situación anterior: el primero son los avances de los SIG, las tecnologías que los soportan y las técnicas de análisis asociadas que operan sobre objetos y campos geográficos continuos y/o discretos, el segundo es el aumento del personal capacitado para el uso de los SIG y el tercero es el incremento de la disponibilidad de cartografía digital y bases de datos geográficas acompañadas de funciones de geo-codificación y georeferenciación [13]; Los hechos anteriores facilitan la utilización de los SIG en salud para describir la magnitud de los problemas, identificar sus factores determinantes, monitorear las desigualdades y generar el conocimiento geográfico para apoyar la toma de decisiones, en este último aspecto las funcionalidades de visualización en mapas temáticos digitales incluidas en los SIG se convierten en una poderosa herramienta para sobrepasar las fronteras geográficas de los tomadores de decisiones limitadas por su alcance visual sobre el horizonte permitiéndoles cubrir extensiones considerables del territorio para facilitar la comprensión de las problemáticas.

Una de las áreas de mayor aplicación de los SIG en salud es en estudios epidemiológicos donde, esta herramienta mediante la aplicación de las técnicas de análisis incorporadas permite la rápida representación en mapas de coropletas de los eventos de salud y sus posibles factores determinantes asociados [14], [15], [16]; por lo general, para este tipo de estudios la priorización y selección de los eventos de interés se hace objetivamente de acuerdo con criterios preestablecidos, uno de los criterios más utilizados para la priorización son las 10 primeras causas individuales de ocurrencia o las cinco primeras causas dentro de cada una de las agrupaciones de causas existentes, dicha selección obedece a que son los eventos más frecuentes en la población.
La ECV por ejemplo, a través del tiempo se ha posicionado dentro de las primeras cinco causas individuales de muerte, lo que la constituye en un problema de atención prioritaria de salud pública. Según la Organización Mundial de la Salud-OMS la ECV es responsable para el año 2008 de alrededor del $11 \%$ de las defunciones mundiales [17], [18], con un incremento de su importancia relativa a través del tiempo, producto del envejecimiento de la población y cambios en estilos de vida como: obesidad, dieta rica en grasas y pobre en fibras, hábito tabáquico e inactividad física. Además, causan en los países desarrollados entre el $10 \%$ y $12 \%$ del total de las muertes, ocupando el tercer lugar como causa de muerte después de la enfermedad coronaria y el cáncer [14]. Además el $88 \%$ de las muertes se presentan en los mayores de 65 años y entre el 10 y el $40 \%$ son muertes pre-hospitalarias [14].

Al igual que la prevalencia, algunos estudios muestran una tendencia clara de disminución anual en la mortalidad por la ECV, comportamiento que se ha observado en diferentes estudios en países desarrollados. Sin embargo, esta tendencia ha disminuido en los últimos años y la curva de descenso de la mortalidad se ha aplanado [19].

Con respecto al riesgo de muerte por ECV se ha evidenciado que es mayor durante el primer mes del evento (17-34\%) y está asociado con la edad, las condiciones de salud de la población y el tipo de ECV. Dicho riesgo además es mayor en los eventos hemorrágicos. En el estudio Rotterdam al final de los primeros 28 días de ocurrido un ataque cerebrovascular, el $12,4 \%$ de los casos isquémicos habían fallecido frente a un $33 \%$ de ataques hemorrágicos, y al cabo del primer año la mortalidad alcanzó el $23.6 \%$ en las ECV isquémicas y el $62.9 \%$ en las hemorrágicas [5]. La mitad de estas muertes son una consecuencia directa de las secuelas neurológicas. En otro estudio que incluyó 978 personas con un primer ECV, el $45.1 \%$ de los casos sobreviven cinco años [6]. Otros estudios como el estudio Framingham, muestran que solo el $35 \%$ de los pacientes sobreviven 10 años [20].

Los estudios anteriores exploran el comportamiento de la ECV mediante el enfoque tradicional sin profundizar en las relaciones espacio-temporales, dicho enfoque ha permitido obtener conocimiento clínico de la enfermedad y adicionalmente de manera individual ha permitido identificar los factores de riesgo asociados y caracterizar la mortalidad ocasionada, no obstante debido primordialmente a que la curva de mortalidad se ha aplanado es necesario complementar su abordaje con otro enfoque que permita evidenciar, además, su comportamiento espacio-temporal para impactar positivamente su ocurrencia. En este sentido a continuación se analizan algunos de los estudios orientados a modelar la mortalidad desde la 
denominada geografía médica a los niveles mundial, europeo, estadounidense y suramericano con énfasis en el territorio colombiano.

En el panorama mundial, la Organización Mundial de la Salud-OMS en el año 2004 elaboró un atlas de enfermedades cardiovasculares y accidentes cerebrovasculares que permite mediante mapas temáticos de coropletas visualizar el número de muertes por ECV y describir el estado de los factores de riesgo asociados en general para las enfermedades del aparato circulatorio [9]. En dicho Atlas se clasifican los países de acuerdo con el número absoluto de muertes y se destacan países como China, la India y la Federación Rusa, es importante resaltar que no se presentan tasas tan solo los años de vida ajustados por discapacidad-AVAD en inglés DALY estandarizados por edad, sin embargo, en el 2011 con datos del 2008 la OMS publicó el Atlas Global Sobre Prevención y Control de Enfermedad Cardiovascular, en dicho Atlas se estimaron las tasas de mortalidad y los AVAD ambos estandarizados por edad para la ECV, los resultados de dicho atlas son presentados en mapas temáticos de coropletas en los que predominan las tasas más altas en Micronesia (Islas Marshall), Asia central(Kirguistán) y Europa Oriental(Federación Rusa y Azerbaiyán) [21].

En el ámbito Europeo para el año 2002, la Oficina Europea de Estadística (Eurostat) desarrolló un Atlas con mapas temáticos de coropletas con las tasas estandarizadas de mortalidad por ECV según sexo para el periodo comprendido entre los años 1994 a 1996, en dicho estudio la ECV se posiciono como la principal causa de muerte en la Unión Europea [22], en el año 1997 en un análisis similar citado por MüllerNordhorn (2008), se estimaron las tasas regionales de mortalidad para los países europeos entre los años 1990-1991 y se analizaron las tendencias de mortalidad para el periodo comprendido entre 1970 y 1992, en dicho estudio se identificó que los países de Europa Occidental presentaban una tendencia hacia tasas de mortalidad inferiores con respecto a la ECV y las tasas más altas de mortalidad las presentaron los países del este y del centro de Europa: Ucrania, Bulgaria y la Federación Rusa, dicho patrón fue confirmado posteriormente por Nordhorn (2008) quien además sugirió clasificar los países del este y del centro de Europa como países de alto riesgo para ECV [23].

A nivel de unidades sub-nacionales de cada país europeo como: cantones (Suiza), departamentos (Francia), provincias(España) o sus equivalentes, en países como España en diferentes estudios se ha explorado a nivel de sus provincias la distribución geográfica de la mortalidad por ECV, en dichos estudios se calcularon las tasas de mortalidad específicas por sexo ajustadas principalmente por edad y se identificó un patrón sistemático donde las tasas más altas, se sitúan claramente en la mitad sur de
España, en Levante y en Galicia [24].

Para la elaboración de los modelos anteriores se utilizaron de manera generalizada: datos de mortalidad por enfermedades cardiovasculares principalmente por ECV, población -censal, estimada y/o proyectada-, mapas digitales a diferentes niveles de desagregación, años de vida perdidos ajustados por discapacidad, tasas de mortalidad estandarizadas ajustadas y las tasas de mortalidad específicas; en los estudios con mapas a niveles de desagregación más detallados se utilizaron los datos disponibles de los registros individuales de defunción y en los más generales se utilizaron datos de las estadísticas disponibles, es importante resaltar que para los modelos anteriores se utilizó principalmente la técnica de análisis espacial básico denominada "análisis de distribución espacial por cartografía temática", mediante la cual los datos de las tasas de mortalidad se representaron en mapas coropléticos clasificados según diferentes criterios.

En Norteamérica, específicamente en Estados Unidos, el Centro para el Control de Enfermedades (CDC) adelantó un estudio entre 1991 y 1998 donde se encontró mediante el uso de mapas temáticos de coropletas elaborados con las tasas y estadísticas de mortalidad por ECV, que para negros y blancos los condados con las mayores tasas de mortalidad de ECV se ubican en los estados del sureste y la región del Delta del Mississippi, además, las tasas según estado variaron de un máximo de 169\%000 en Carolina del Sur a un mínimo de 89\%000 en Nueva York [25]. En el año 2011, en otro estudio realizado por el CDC y mediante el uso de SIG, específicamente mediante consultas de selección de áreas prioritarias se encontró que la más alta prevalencia $(\geq 11 \%)$ de la diabetes está ubicada en del denominado "cinturón de la diabetes", que tiene una amplia coincidencia con el cinturón de $E C V$, dicha zona geográfica incluye un área comprendida por 644 condados de 15 estados [26].

A nivel de Sur América desde el Centro de Investigaciones Geográficas (FCH-UNICEN), y como parte de la experiencia de trabajo conjunto con la municipalidad de Tandil en Argentina, el proceso automático de geo-referenciación en mapas temáticos y procesamiento de eventos vitales se convierte en un insumo fundamental para los estudios epidemiológicos [27]. En Chile también existen iniciativas para implementar SIG en salud [28], específicamente para ECV en el año 2005 se elaboró el atlas de mortalidad para enfermedades isquémicas y enfermedades cerebrovasculares entre 1997 y 2003, en dicho atlas se estimaron las tasas de mortalidad a nivel de las 339 comunas evidenciándose problemas en la estimación de las tasas en las comunas con un numero de eventos por debajo de 20 defunciones; para la representación de las tasas se utilizaron mapas temáticos de coropletas que permiten evidenciar la distribución geográfica para cada comuna evidenciándose que las 
comunas con tasas más altas se ubican en la regiones Bío-Bío y Araucanía [29].

Según las evidencias anteriores es importante concluir que en general las técnicas utilizadas para explorar el comportamiento espacio-temporal en los estudios revisados, aún no incluyen análisis espaciales avanzados sobre múltiples variables en periodos de tiempo prolongados y a niveles de desagregación detallados, es decir, los análisis aún son análisis básicos que permiten una aproximación inicial a la comprensión del comportamiento espacio-temporal la ECV.

En Colombia, particularmente, "la población ha experimentado un proceso de transición demográfica caracterizado por un descenso de la fecundidad y mortalidad, lo que ha conducido a una transición epidemiológica donde las enfermedades crónicas y degenerativas prevalecen frente a las infecciosas" [30], tal es el caso de las enfermedades crónicas del aparato circulatorio y específicamente de la ECV donde según estudios recientes con agrupación de causas de muerte según las listas 6/67 y 6/61 de OPS, se identificó en primer lugar al grupo de enfermedades del aparato circulatorio; además de una tendencia a la disminución en las tasas de mortalidad para dicho grupo en el periodo comprendido entre 1987 y el 2008 con tasas que oscilan entre un valor mínimo de $131,8 \% \%_{000}$ y un valor máximo de $180,9 \%$ o00; también se logró identificar que las tasas más altas se presentan principalmente en los hombres adultos mayores y se agrupan geográficamente en la región andina [31], [32], [33], [34], [35], [36], este último hallazgo fue confirmado en el estudio desarrollado por Zapata (2012) donde mediante el uso de SIG y la aplicación de un análisis de clúster se identificó para las enfermedades del aparato circulatorio un clúster alto que se correlaciona positivamente con las tasas de sus vecinos en la región Andina con tendencia a concentrarse en el centro del país [7], los resultados sugieren que el comportamiento de la ECV debe ser muy similar al que se evidencia en el grupo en general pero dicha hipótesis aún no está comprobada. También se ha identificado mediante el uso de SIG que el grupo de las enfermedades del sistema circulatorio fue el grupo de causas que presentó la mayor pérdida de AEVG y que el $77,8 \%$ de los municipios de Colombia perdieron en promedio 2,15 AEVG, de los cuales el 9,0\% perdieron más de 5 años de esperanza de vida y se identificó que los municipios que presentaron una mayor pérdida para este grupo de enfermedades se ubican en: Boyacá, Huila y Santander [37]. Específicamente, para la mortalidad por ECV las cifras más recientes aún sin consolidar según el DANE refieren que la mortalidad causada por la ECV para el año 2011 ocupa el tercer lugar con un total de 13.611 muertes registradas.

Es importante resaltar que en el contexto latinoamericano Colombia se ha posicionado como uno de los países pioneros en el estudio epidemiológico de la ECV [38] mediante diferentes aproximaciones metodológicas que incluyen estudios multi-céntricos para obtener evidencia acerca del impacto de los factores de riesgo clásicos y emergentes e investigaciones a nivel municipal como: el estudio sobre la epidemiología de la ECV en SabanetaAntioquia [39] y los estudios desarrollados en otros municipios como Jamundí, Girón y Pie de cuesta. No obstante, los hallazgos actuales no son suficientes para intervenir e impactar positivamente la problemática y se hace necesario el desarrollo de nuevos estudios que utilicen los servicios ofrecidos por los SIG para caracterizar la ECV, identificar su comportamiento espacio-temporal y contribuir explicar las diferencias a nivel municipal en la población colombiana. Estas diferencias podrían estar asociadas a diferentes factores de riesgo tanto tradicionales como emergentes, así como a factores sociales, ambientales y posiblemente genéticos y solo con la profundización sobre dichos aspectos, se permitirá el abordaje desde la perspectiva de prevención primaria y secundaria, para contribuir al diagnóstico temprano, disminuir la discapacidad, asegurar el acceso a formas efectivas de tratamiento y garantizar la rehabilitación en las personas afectadas con el fin de impactar positivamente su ocurrencia.

Al analizar comparativamente los modelos espaciotemporales utilizados para estudiar la mortalidad por ECV se pueden resaltar los siguientes aspectos relacionados con sus componentes: en cuanto a los datos de entrada utilizados, todos utilizan datos geográficos y no geográficos similares entre los que se pueden destacar mapas digitales del área de estudio global y de la unidad de desagregación sub-nacional requerida por cada estudio, datos de: población censal, proyectada y/o estimada- años de vida perdidos ajustados por discapacidad, tasas ajustadas de mortalidad estandarizadas y solo en casos puntuales el valor absoluto del número de muertes, esto debido a que dichos valores no son comparables, con respecto a este componente es importante resaltar que para utilizar modelos avanzados de análisis espacio-temporal se requiere además de los datos anteriores disponer de datos sobre la mortalidad por ECV en diferentes series temporales y de los factores de riesgo geo-referenciados en las unidades subnacionales propias del estudio. En cuanto al procesamiento y análisis de los datos de manera general los modelos utilizados no permiten valorar conjuntamente el comportamiento en el espacio y el tiempo, tan solo se limitan a representarlos por separado, adicionalmente se utilizan de manera generalizada los análisis de representación mediante mapas temáticos de coropletas, dichos análisis corresponden a la técnica "análisis de distribución espacial por cartografía temática", comparativamente los elementos que cambian de un modelo a otro solo corresponden al criterio utilizado para la agrupación de las categorías donde predomina el uso de cuartiles. El último aspecto comparado fueron las salidas, al respecto se encontró que como salida generalizada de 
todos los modelos de los estudios revisados, se obtienen mapas coropléticos acompañados de tablas estadísticas y la única diferencia radica en el tipo mapa temático empleado, debido a que en casos puntuales como el del Atlas de Enfermedades Cardiovasculares y ECV de la OMS (2004), se usaron cartodiagramas con datos complementarios en gráficos de pastel ilustrativos. Como se puede evidenciar en cuanto al uso de modelos espacio-temporales queda un amplio camino por recorrer, pero se debe reconocer que el uso de indicadores de salud y bienestar estimados rigurosamente, a escalas geográficas diversas posibilita alcanzar las directrices de la Unión Geográfica Internacional con respecto al uso de la geografía en salud para profundizar sobre las relaciones entre la salud medioambiente, recursos naturales y urbanización.

\section{MODELO PARA EL ESTUDIO DE LA MORTALIDAD ECV}

Con el fin de contribuir al desarrollo de trabajos futuros sobre la mortalidad por ECV y como aporte final a continuación se propone un modelo general (Figura 1) que integra ambos enfoques -tradicional y espacio temporal- en dicho modelo se incluyen los indicadores de salud y los mapas temáticos detallados y multitemporales, dichos insumos se deben consolidar en una geodatabase cuyos campos y objetos geográficos se definen mediante el conocimiento aportado por ambos enfoques, el enfoque tradicional permite identificar el comportamiento de la mortalidad por ECV según variables individuales y sus factores de riesgo asociados, mientras que el enfoque espaciotemporal permite obtener series temporales, datos geográficos de los factores de riesgo previamente identificados mediante el enfoque tradicional y permite la estimación de indicadores de salud a escalas geográficas diversas. Para el procesamiento de los datos en el modelo se propone el uso de técnicas de estadística multivariada -Análisis de componentes principales, análisis del factor y análisis del discriminante entre otros- combinadas técnicas de inteligencia artificial redes neuronales, algoritmos genéticos y lógica difusa entre otras- y finalmente combinadas con técnicas de análisis espacial básicas y avanzadas y geo-estadística -Análisis multi-criterio, Kriging, Auto-correlación espacial, Análisis de cluster y Regresión Espacial entre otros-. Como salidas generales del modelo se sugieren mapas temáticos de diferentes tipos, entre los que se recomienda el uso de cartodiagramas y mapas temáticos de coropletas que permitan a los tomadores de decisiones ubicar espacial y temporalmente el comportamiento de la mortalidad por ECV. Es importante resaltar que dicho modelo es adaptable para el estudio de otros fenómenos y permite la integración de los dos enfoques utilizados en la actualidad pero de manera aislada. Cada vez que sea usado el modelo se sugiere que los resultados sean divulgados y socializados con los tomadores de decisiones para garantizar el impacto esperado sobre la mortalidad.

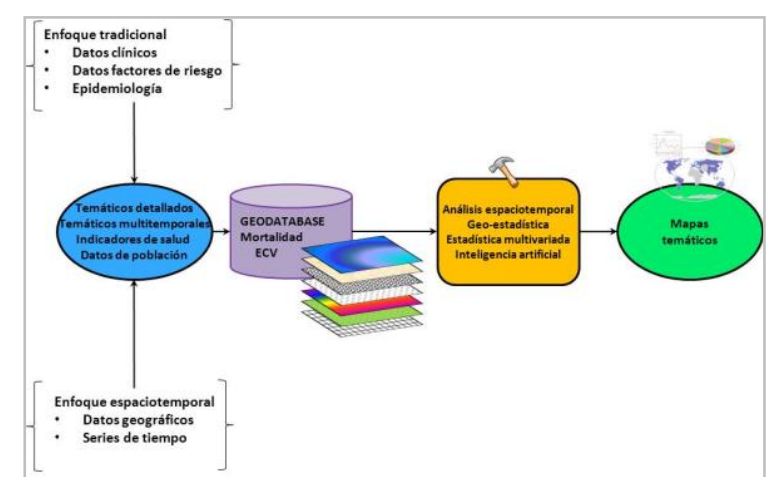

Fig. 1. Modelo para el estudio de la mortalidad por ECV. Fuente: Elaboración propia

A manera de ejemplo se presentan a continuación dos casos de aplicación del modelo representados mediante la herramienta Model Builder del software Arcgis 10, el primer caso está orientado a la modelación mediante un análisis de auto-correlación espacial y el segundo caso considera un análisis de clúster espacial.

Para el primer caso de análisis de auto-correlación espacial, se deben obtener los siguientes datos de entrada: mapas temáticos digitales a nivel municipal, datos de población por municipio, indicadores de mortalidad por ECV como: tasas de mortalidad estandarizadas, años de vida perdidos ajustados por discapacidad y datos geográficos de los factores de riesgo relevantes para ECV a nivel municipal; con el fin de facilitar los procesos de administración de los datos se deben consolidar en una base de datos espacial Geodatabase-, luego los datos deben ser sometidos al proceso de análisis de correlación mediante la herramienta de análisis de patrones de las herramientas estadísticas y finalmente después del procesamiento se obtienen los resultados en tablas y gráficos que soportan el análisis.

En el segundo caso los datos de entrada serían los mismos pero para el procesamiento se debe utilizar la herramienta Anselin Local Moran's I, mediante la cual se obtienen mapas temáticos con: las medidas de significancia estadística (puntuaciones de $Z$ con sus respectivos valores de probabilidad), las cuales indican si se debe rechazar o no la hipótesis nula de que los valores asociados con entidades están distribuidos en forma aleatoria, también se obtienen los valores del índice I local de Moran y el tipo de clúster; según los valores obtenidos del índice I local de Moran se sugiere clustering cuando los valores sean positivos 0 dispersión (las entidades no hacen parte de un clúster) cuando los valores obtenidos sean negativos, adicionalmente el valor obtenido para el tipo de cluster permite distinguir entre un cluster de valores altos (AA), un cluster de valores bajos (BB) y cluster de valores atípicos ( $\mathrm{AB})$ o (BA). A continuación se presentan ambos modelos en las Figuras 2 y 3 respectivamente. 


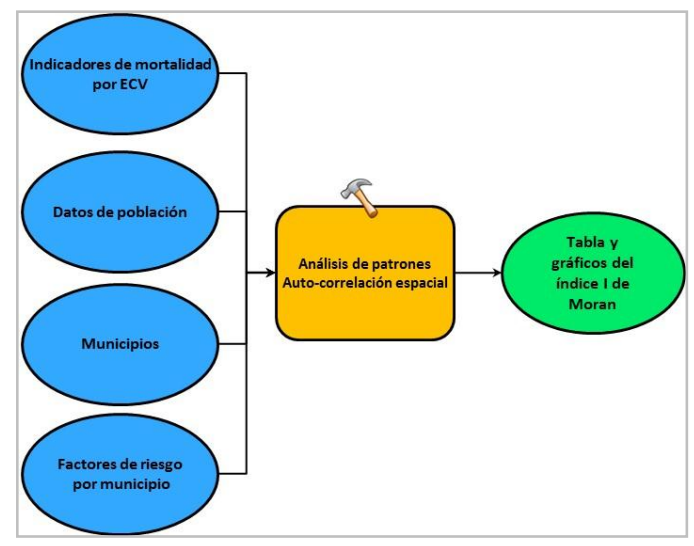

Fig. 2. Modelo análisis de auto-correlación de la mortalidad por ECV.

Fuente: Elaboración propia

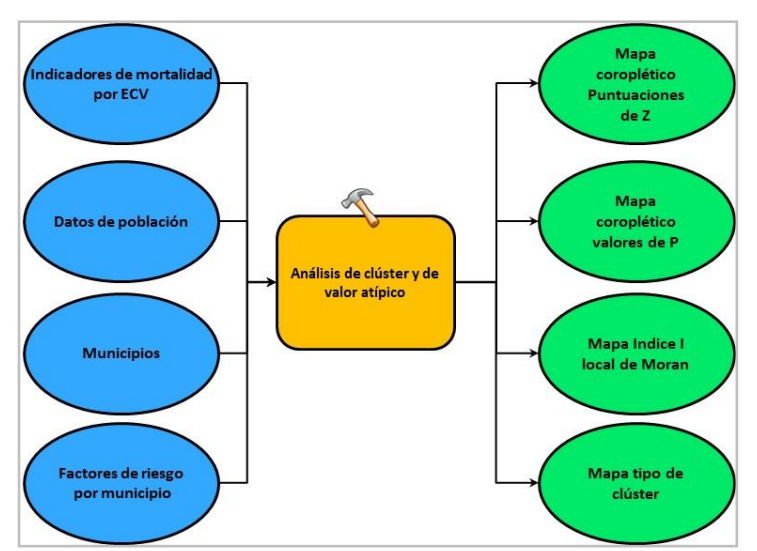

Fig. 3. Modelo análisis de clúster y de valor atípico de la

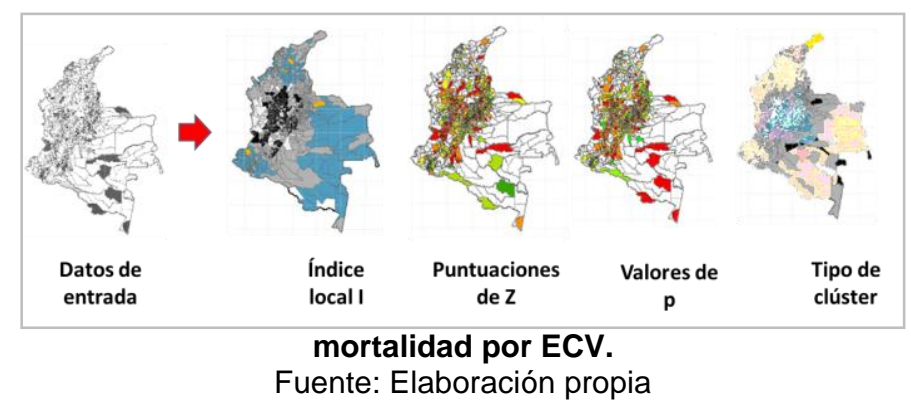

El primer modelo de ejemplo (Figura 2) permite identificar si existe auto-correlación espacial es decir, si el patrón de comportamiento de la mortalidad por ECV esta agrupado, disperso o es aleatorio, adicionalmente, el segundo modelo (Figura 3), derivado del modelo de análisis general permite identificar los puntos calientes, puntos fríos y valores atípicos espaciales estadísticamente significativos para la mortalidad por ECV. Es importante resaltar que el modelo general permite además utilizar otras herramientas más robustas para modelar la mortalidad por ECV con el fin de apoyar la toma de decisiones y soportar objetivamente el establecimiento de políticas.

\section{TRABAJOS FUTUROS}

En el futuro cercano se desarrollaran proyectos de investigación orientados a documentar el estado del arte de la mortalidad por ECV en Colombia y explorar las relaciones, variaciones y patrones espacio- temporales de la ECV a nivel municipal mediante un enfoque integrado que utilice el modelo en pleno, además se exploraran los posibles factores asociados con el fin de retroalimentar las políticas nacionales, reorientar las acciones y finalmente establecer un sistema de vigilancia epidemiológica enmarcado dentro de un observatorio activo de la ECV en el territorio colombiano.

\section{CONCLUSIONES}

En una ventana de tiempo conformada por los últimos 15 años a nivel mundial, los dos enfoques -tradicional y espacio-temporal- identificados para el estudio de la mortalidad por ECV permiten de manera aislada su caracterización a niveles de desagregación generales, pero poco se ha explorado a niveles de desagregación detallados como los condados, las parroquias, las comunas o los municipios en el caso colombiano, además, se evidencia que para la modelación de la mortalidad por ECV con el enfoque espacio-temporal, en los estudios revisados comparativamente se utilizan datos similares y técnicas básicas de análisis espacial y en un caso puntual para el grupo de enfermedades del aparato circulatorio sin particularizar el comportamiento para la ECV, se utilizó un análisis de clúster considerado una de las técnicas avanzadas; también es evidente que se carece de aproximaciones que combinen los dos enfoques e implementen técnicas avanzadas de estadística multivariada, inteligencia artificial, geo-estadística y análisis espacial, por lo tanto, se propone un modelo de análisis que implementa técnicas avanzadas y además incluye ambos enfoques integrados, los objetivos principales de dicho modelo son consolidarse como un referente para el estudio de la mortalidad por ECV e impactarla positivamente mediante la generación del conocimiento requerido para soportar: la toma de decisiones informada, el establecimiento de políticas, la reorientación objetiva de las acciones y la optimización de los recursos.

\section{REFERENCIAS}

[1] J. Mackay and G. Mensah, eds. "The Atlas of Heart Disease and Stroke", World Health Organization, Geneva, 2004.

[2] A. Arana, C. S. Uribe, A. Muñoz, F. A. Salinas and J. I. Celis, "Guías de Práctica Clínica Basadas en la Evidencia". Enfermedad Cerebrovascular. 2000:41.

[3] I. H. Burnley and D. Rintoul, "Inequalities in the transition of cerebrovascular disease mortality in New South Wales, Australia 1969-1996". Soc Sci Med., 54(4), pp. 545-59, 2002.

[4] N. Yiannakoulias, M. D. Hill and L. W. Svenson, "Geographic hierarchies of diagnostic practice style in cerebrovascular disease". Social Science \& Medicine, 68(11), pp.1985-92, 2009.

[5] M. Hollander, P. Koudstaal, M. Bots, D. Grobbee, A. Hofman and M. Breteler, "Incidence, risk, and case fatality of first ever stroke in the elderly population. The Rotterdam Study". J Neurol Neurosurg Psychiatry, 74, pp. 317-321, 2003. 
[6] P. Seana, J. W. Sturm, H. M. Dewey, G. A. Donnan, R. Macdonell and A. Thrift, "Long-term outcome in the north east Melbourne stroke incidence study predictors of quality of life at 5 years after stroke". Stroke:36, pp. 2082-2086, 2005.

[7] S. Zapata, "Estructura de la mortalidad por causa, según municipio y su relación espacial con algunas condiciones socioeconómicas, Colombia 1993 y 2005". Medellín: Universidad de Antioquia, Facultad Nacional de Salud Pública. 2012.

[8] C. A. Tangarife et al, "Análisis de situación de salud de Colombia 2002-2007: Universidad de Antioquia", Facultad Nacional de Salud Pública. Gestión de datos. 2010. [Online].

[9] P. Elliott, J. Wakefield, N. Best and D. Briggs, "Disease mapping: a historical perspective". In: Spatial epidemiology: methods and applications. Oxford University Press: 223-239. 2000.

[10] W. SD and B. SE. "Mapping mortality and morbidity patterns: an international comparison", International Journal of Epidemiology, 20(3): pp. 678-89, 1991.

[11] G. López-Abente and C. Ibáñez, "Aplicación de técnicas de análisis espacial a la mortalidad por cáncer en Madrid”. Documentos Técnicos de Salud Pública. № 66 . CONSEJERIA DE SANIDAD. Dirección General de Salud Pública. Comunidad de Madrid. España, 2001.

[12] E. Loyola, C. Castillo-Salgado, P. Nagera-Aguilar, M. Vidaurre, O. J. Mujica and R. Martínez-Piedra, "Los sistemas de información geográfica como herramienta para monitorear las desigualdades de salud". Revista Panamericana de Salud Pública/ Panamerican Journal of Public Health, 12(6): pp. 415-428, 2002.

[13] Bonita R. "Epidemiology of Stroke". Lancet, pp. 344-349, 1992.

[14] Organización Panamericana de la Salud. Programa Especial de Análisis de Salud. Sistemas de información geográfica en salud: conceptos básicos. $1^{\underline{a}}$ Ed. Organización Panamericana de la Salud, 2002. p.112. ISBN: 9789275323427.

[15] C. A. Tangarife, "Sistemas de información geográfica y su aplicación en salud pública". Memorias VIII curso internacional: Eco-epidemiología de la enfermedad de chagas y métodos para su estudio, pp.35-51, 2005.

[16] World Health Organization, "WHO Global InfoBase Online", Noviembre 2012. [Online]. Available: www.who.int

[17] World Health Organization, "The top ten causes of death". Online [Noviembre 2012].

[18] M. T. Olalla, M. J. Medrano and J. Almazán, "Mortalidad por Enfermedad Cerebrovascular en España". Rev. Neurol, 29: p. 872, 1999.

[19] L. H. Kuller, "Epidemiology and prevention of stroke, now and in the future". Epidemiol Reviews, 22:14-7, 2000.

[20] S. Mendis, P. Puska and B. Norrving (editors), "Global Atlas on cardiovascular disease prevention and control", ISBN 978-92-4-156437-3, 2011.

[21] Eurostat, "Atlas de mortalidad: enfermedades cardiovasculares, capítulo 8". Online [Enero 2013].

[22] J. Müller-Nordhorn, S. Binting, S. Roll and S. N. Willich. "An update on regional variation in cardiovascular mortality within Europe". European Heart Journal. Online [2008].

[23] Fundación Española del Corazón. "Informe de la salud cardiovascular en España en el contexto europeo". Online [2008].
[24] CDC. "Releases Atlas of Stroke Mortality, Centers for Disease Control and Prevention, National Center for Chronic Disease Prevention \& Health Promotion", Online [Feb. 2003].

[25] L. Barker, K. Kirtland, E. Gregg, L. Geiss, T. Thompson, "Geographic distribution of diagnosed diabetes in the United States: a diabetes b. American Journal of Preventive Medicine 2011". Online [Nov. 2012].

[26] S. Linares, A. Tisnés and A. Nochera, "Aplicación de sistemas de información geográfica para detectar patrones espaciales de la mortalidad por cáncer en la ciudad de Tandil, en Geografía Argentina: aportes al estudio de algunos problemas actuales". CIG. Facultad de Ciencias Humanas. Universidad Nacional del Centro de la Provincia de Buenos Aires. ISBN 978-950-658205.-0. 2008.

[27] D. Rodríguez, E. Salinas and X. Aguilera, "Análisis espacial de la difusión de la hepatitis A en Chile" Boletín Electrónico Mensual de Vigilancia Epidemiológica. Ministerio de Salud, Departamento de Epidemiología. Boletín № 31, 2004. Online [Oct. 2012].

[28] M. G. Icaza and M. L. Nuñez, "Atlas de mortalidad por Enfermedades Cardiovasculares en Chile, 1997-2003". Universidad de Talca. 2003.

[29] Ministerio de Protección Social. Universidad Nacional de Colombia. Situación de salud en Colombia 2005. Informe Preliminar.

[30] A. Vidal, "Mortalidad por causa básica de defunción, sexo, lugar de residencia, edad y transferencias de recursos a los municipios. Colombia 2007-2008". Medellín: Universidad de Antioquia, Facultad Nacional de Salud Pública. 2011.

[31] A. Espinosa. "Mortalidad por causa básica de defunción, sexo, lugar de residencia, edad y transferencias de recursos a los municipios. Colombia 1999-2002". Medellín: Universidad de Antioquia, Facultad Nacional de Salud Pública. 2012.

[32] Osorio A. "Mortalidad por causa básica de defunción, sexo, lugar de residencia, edad y transferencias de recursos a los municipios. Colombia 1995-1998". Medellín: Universidad de Antioquia, Facultad Nacional de Salud Pública. 2012.

[33] L. Dávila, "Mortalidad por causa básica de defunción, sexo, lugar de residencia, edad y transferencias de recursos a los municipios. Colombia 1987-1990". Medellín: Universidad de Antioquia, Facultad Nacional de Salud Pública. 2012.

[34] T. Contreras, "Mortalidad por causa básica de defunción, sexo, lugar de residencia, edad y transferencias de recursos a los municipios. Colombia 1991-1994". Medellín: Universidad de Antioquia, Facultad Nacional de Salud Pública. 2012.

[35] I. Muriel, "Mortalidad por causa básica de defunción, sexo, lugar de residencia, edad y transferencias de recursos a los municipios. Colombia 2003-2006". Medellín: Universidad de Antioquia, Facultad Nacional de Salud Pública. 2012.

[36] A. Toro, "Comportamiento de la mortalidad por causa básica de defunción y su contribución al cambio en la esperanza de vida por municipio de residencia en Colombia 1992-1994 y 2004-2006". Medellín: Universidad de Antioquia, Facultad Nacional de Salud Pública. 2012. 
[37] F. A. Silva, J. G. Zarruk, C. Quintero, W. Arenas, C. F. Rueda-Clausen, S. Y. Silva et al. "Cerebrovascular disease in Colombia". Rev. Col. Cardiol.; 13(2): pp. 8589. Online [Enero 2013].
[38] C. S. Uribe, I. Jimenez, M. O. Mora, A. Arana, J. L. Sánchez, L. Zuluaga, et al. "Epidemiología de las enfermedades cerebrovasculares en Sabaneta, Colombia (1992-1993)". Rev Neurol, 25: pp. 1008-1012, 1997. 